E. Yu. Romanenko, M. B. Vereikina, cand. phys.-math. sci. (Inst. Math. Ukr. Acad. Sci., Kiev)

\title{
ON SIMULATION OF SPATIAL-TEMPORAL CHAOS: THE SIMPLEST MATHEMATICAL PATTERNS AND COMPUTER GRAPHICS*
}

\section{МОДЕЛЮВАННЯ ПРОСТОРОВО-ЧАСОВОГО ХАОСУ: НАЙПРОСТІШІ МАТЕМАТИЧНІ МОДЕЛІ TА КОМП'ЮТЕРНА ГРАФІКА*}

For a certain nonlinear boundary-value problem for PDE, the article represents three scenarios for evolution of spatial-temporal chaos and specifies the corresponding types of chaotic solutions. Analytical assertions are illustrated by numerical analysis and computer graphics.

Для однієї граничної задачі для рівнянь у частинних похідних розглядаються три випадки еволюції просторово-часового хаосу і визначаються відповідні типи хаотичних розв'язків. Аналітичні твердження супроводжуються ілюстраціями, одержаними за допомогою комп'ютерного експерименту та машинної графіки.

1. Introduction. In the whole world, scientists attach much importance to nonlinear dynamics dealing with two (interconnected) trends in the evolution of nonlinear systems: self-structure and chaotization. At present, a certain progress in clarifying the nature of the so-called dynamical chaos has been achieved. By the dynamical chaos, we mean a transition from a regular behavior to the chaotic one in a deterministic system (i.e., in a system, whose initial state and state dynamics obey deterministic laws). Lots of works in this field are based on the strange-attractor theory and examine, mainly, the chaos of simplest type, i.e., the temporal chaos, which shows the quasirandom behavior of a system in time (i.e., the nonregularity of the state evolution for large time (see, e.g., [1])). The certain hopes are set on the strange-attractor concept in connection with investigation of the spatial-temporal chaos, for which not only the temporal behavior of a system but also the spatial one is quasirandom (i.e., when time is large, both system states (in space) and their evolution are nonregular). This approach (from the viewpoint of the strange-attractor theory) usually assumes approximating real systems by finite-dimensional dynamical systems.

The next step on the way of constructing the spatial-temporal chaos theory is the method of coupled map lattice (i.e., "countable-dimensional" dynamical systems), which has appeared relatively recently (see, e.g., [2]).

The present work uses the approach [3], alternative to these methods, which is based on the simulation of spatial-temporal chaos by infinite-dimensional nonlinear dynamical systems. The gist of this approach consists in the reduction of a corresponding mathematical pattern to studying the dynamics of a continual family of uncoupled (or weakly coupled) maps.

The paper seeks to exhibit a wide scope for investigating the dynamical chaos by means of the "continual" approach.

The simplest mathematical patterns for deterministic evolutionary systems are given by nonlinear boundary-value problems for linear hyperbolic PDE of the form

$$
\begin{gathered}
\frac{\partial u_{i}}{\partial t}+\sum_{j=1}^{m} a_{i j} \frac{\partial u_{i}}{\partial x_{j}}=0, \quad x=\left(x_{1}, \ldots, x_{m}\right) \in G \subset \mathbb{R}^{m}, \\
\left.H\left(u, \frac{\partial u}{\partial t}, \frac{\partial u}{\partial x}\right)\right|_{\partial G}=0, \quad u=\left(u_{1}, \ldots, u_{n}\right),
\end{gathered}
$$

where $a_{i j}$ is a constant $m \times n$ matrix and $H: \mathbb{R}^{n+m} \mapsto \mathbb{R}$ is a nonlinear function. An important advantage of this kind of problems is the following: On the one hand, we

\footnotetext{
* Partially supported by Fund for fundamental research of the State Committee of Ukraine on Science and Technology.
} 
can analyze them in detail (and, in addition, comparatively simply) and, on the other hand, although the problems reflect reality in a very abstract (ideal) form, they make it possible to reveal the mathematical mechanism of self-initiating and evolution of spatial-temporal chạotization in real systems. The problems of form (1) can be effectively investigated, first, because of their reducibility to difference equations (this fact was noted in the thirties, e.g., in [4]) and, second, owing to the achievements of the modern theory of dynamical systems and the theory of difference and differentialdifference equations (this class of problems was discussed in [3] in detail). By taking a specific problem of form (1) as an example, we consider the "continual" method and examine some scenarios of forming the spatial-temporal chaos from a regular initial state under a deterministic evolution law.

2. The statement of the problem. We consider the simplest of the multidimensional problems (1), namely, the linear system of two (uncoupled) equations with two spatial variables

$$
\begin{aligned}
& \frac{\partial u}{\partial t}=a_{11} \frac{\partial u}{\partial x}+a_{12} \frac{\partial u}{\partial y}, \\
& \frac{\partial v}{\partial t}=a_{21} \frac{\partial v}{\partial x}+a_{22} \frac{\partial v}{\partial y} .
\end{aligned}
$$

with the nonlinear boundary conditions

$$
\begin{gathered}
{\left.[u-v]\right|_{y=0}=0,} \\
{\left.\left[F_{1}(u, v) u_{t}^{\prime}+F_{2}(u, v) v_{t}^{\prime}\right]\right|_{y=1}=0}
\end{gathered}
$$

and with the initial conditions

$$
\begin{aligned}
& \left.u\right|_{t=0}=u_{0}(x, y), \\
& \left.v\right|_{t=0}=v_{0}(x, y) .
\end{aligned}
$$

If the functions $F_{1}$ and $F_{2}$ are continuous, then the left-hand side of equation (4) can always be reduced to the total differential $d F(u, v)=0$ (by means of multiplying the equation by an integrating factor). Thus, we can transform boundary condition (4) to the form $\left.F(u, v)\right|_{y=1}=\lambda(x)$, where $\lambda(x)$ is an arbitrary function.

We seek a solution of problem $(2)-(5)$ in the class $C^{1}(G \times[0, T], \mathbb{R}), T \leq+\infty$. This imposes the following additional restrictions on the problem:

1) $u_{0}$ and $v_{0}$ are $C^{1}$-functions; $F_{1}$ and $F_{2}$ are $C^{0}$-functions;

2) the initial data are in agreement with the boundary ones, i.e., $u_{0}(x, 0)=v_{0}(x, 0)$, $\lambda(x)=F\left(u_{0}(x, 1), v_{0}(x, 1)\right)$. Consequently, on the boundary $y=1$, condition (4) takes the form

$$
\left.F(u, v)\right|_{y=1}=\lambda_{0}(x)
$$

where $\lambda_{0}(x)=F\left(u_{0}(x, 1), v_{0}(x, 1)\right)$.

Moreover, we demand that the correctness conditions (i.e., the conditions, under which the problem (2)-(5) has a unique $C^{1}$-solution) be satisfied. The necessary (but not sufficient) conditions for the correctness follows directly from the technique of solving problem (2) $-(5)$ by the method of characteristics:

1) $a_{12} a_{22}<0$

$2)$ in the plane $(u, v)$, the points with the coordinates $u=u_{0}(x, 1), v=v_{0}(x, 1)$, 
$x \in \mathbb{R}$, are regular for the vector field $\left(F_{1}, F_{2}\right)$ (this is true, obviously, when $F_{i}\left(u_{0}(x\right.$, 1), $\left.\left.v_{0}(x, 1)\right) \neq 0, i=1,2, x \in \mathbb{R}\right)$.

Under the same assumptions, the specific problem of form (2)-(5) was considered in [3].

The idea of investigation consists in reducing problem (2)-(5) to a difference equation. In the class $C^{1}$, the general solution of system (2) is represented as the travelling waves

$$
\begin{aligned}
& u(x, y, t)=\varphi\left(x+a_{11} t, y+a_{12} t\right), \\
& v(x, y, t)=\psi\left(x+a_{21} t, y+a_{22} t\right),
\end{aligned}
$$

where $\varphi$ and $\psi$ are arbitrary $C^{1}$-functions. Conditions (3), (6), and (5) give unique functions $\varphi$ and $\psi[4,5]$, namely

$$
\begin{gathered}
\varphi(z, \tau)=w(z, \tau), \\
\psi(z, \tau)=w(z-b \tau, \tau-c \tau), z \in \mathbb{R}, \tau \in[1-c,+\infty),
\end{gathered}
$$

where $b=\left(a_{21}-a_{11}\right) / a_{22}, c=1-a_{12} / a_{22}(c>0)$, and $w(z, \tau)$ is the solution of the difference equation

$$
F(w(z+b, \tau+c), w(z, \tau))=\lambda\left(z-\frac{a_{11}}{a_{12}} \tau+\frac{a_{21}}{a_{22}}\right)
$$

with the initial condition

$$
\begin{gathered}
\left.w(z, \tau)\right|_{(z, \tau) \in \mathbb{R} \times[1-c, 1)}=w_{0}(z, \tau)= \\
=\left\{\begin{array}{l}
v_{0}\left(z+\frac{b}{1-c} \tau, \frac{1}{1-c} \tau\right), z \in \mathbb{R}, \tau \in[1-c, 0), \\
u_{0}(z, \tau), z \in \mathbb{R}, \tau \in[0,1) .
\end{array}\right.
\end{gathered}
$$

Consequently, the solution $(u, v)$ of problem (2)-(5) can be written in terms of difference problem (9)-(10) as follows

$$
\begin{gathered}
u(x, y, t)=w\left(x+a_{11} t, y+a_{12} t\right), \\
v(x, y, t)=w\left(x-b y+a_{11} t, y-c y+a_{12} t\right) .
\end{gathered}
$$

Note that the investigation of equation (9) can be essentially simplified if the equation is resolved for $w(z, \tau)$ or $w(z+b, \tau+c)$. In the first case, we have an advanced difference equation; in the second case, we have a retarded one.

The reduction carried out above enables us to ascertain a special feature of problems of form (2)-(5). The right-hand side of equation (9) depends on the initial data $u_{0}, v_{0}$. Generally speaking, this may have a strong impact on the asymptotic behavior of the solution $(u, v)$. This means that systems simulated by boundary-value problems of form (2)-(5) do not possess the property of "forgetting initial data", which is typical for many systems with structural turbulence.

3. The simulation of the processes of forming structures and chaos emergence. Below, we investigate a specific problem of form (2)-(5) by the reduction method and consider what types of oscillatory solutions are possible when time is large and how the oscillation behavior depends on the variation of initial data. In particular, the initial data, which generate the solutions that simulate the formation of structures and the emergence of spatial-temporal chaos, are specified.

So, consider the deterministic system such that its state is presented by the vector field $(u(x, y, t), v(x, y, t)),(x, y) \in G=\mathbb{R} \times[0,1]$ at every moment $t=t^{*}$ and the 
dynamics of states (in time) is represented by the nonlinear boundary-value problem

$$
\begin{gathered}
\frac{\partial u}{\partial t}=\frac{\partial u}{\partial x}+\frac{\partial u}{\partial y}, \\
\frac{\partial v}{\partial t}=-\frac{\partial v}{\partial x}-\frac{\partial v}{\partial y}, \quad(x, y) \in G, t \in \mathbb{R}^{+}, \\
{\left.[u-v]\right|_{y=0}=0,} \\
{\left.\left[\left(1-v^{2}\right) u_{t}^{\prime}+2 v(1+u) v_{t}^{\prime}\right]\right|_{y=1}=0,} \\
\left.u\right|_{t=0}=u_{0}(x, y)=\frac{A}{1-A} y\left(1-A \sin ^{2} 2 \pi x\right), \\
\left.v\right|_{t=0}=v_{0}(x, y)=A y \sin 2 \pi x,
\end{gathered}
$$

where $A \in(0,1 / 2]$ is a parameter.

Proceeding like in subsection 2 , we transform boundary condition (13) to the total differential $\frac{d}{d t} \frac{u+1}{1-v^{2}}=0$ by using the integrating factor $p(v)=\left(1-v^{2}\right)^{-2}$. Then (13) takes the equivalent form $\left.\left[u+1=\lambda(x)\left(1-v^{2}\right)\right]\right|_{y=1}$, where $\lambda(x)$ is an arbitrary function of $x$. The initial data yield

$$
\lambda(x)=\frac{1+u_{0}(x, 1)}{1-v_{0}^{2}(x, 1)}=\frac{1}{1-A},
$$

and, consequently, the second boundary condition (14) is replaced by the following

$$
\left.u\right|_{y=1}=\frac{1}{1-A}\left(1-\left.v^{2}\right|_{y=1}\right)-1
$$

and problem (12)-(15) is reduced to problem (12), (13), (16), and (15). The latter is correct and, as has been shown in subsection 2 , can be reduced, in its turn, to the difference problem

$$
\begin{gathered}
w(z+2, \tau+2)=\frac{1}{1-A}\left(1-w^{2}(z, \tau)\right)-1, \quad z \in \mathbb{R}, \tau \in[1-c,+\infty), \\
\left.w(z, \tau)\right|_{(z, \tau) \in \mathbb{R} \times[1-c, 1)}=w_{0}(z, \tau)= \\
=\left\{\begin{array}{l}
v_{0}(z-2 \tau,-\tau), \quad z \in \mathbb{R}, \tau \in[1-c, 0), \\
u_{0}(z, \tau), \quad z \in \mathbb{R}, \tau \in\left[\begin{array}{ll}
0, & 1),
\end{array}\right.
\end{array}\right.
\end{gathered}
$$

by using the formulas (in other words, the change of variables)

$$
\begin{gathered}
u(x, y, t)=w(x+t, y+t), \\
v(x, y, t)=w(x-2 y+t,-y+t) .
\end{gathered}
$$

Let $f_{A}(w):=(1-A)^{-1}\left(1-w^{2}\right)-1, w \in[-1,1]$ and $t=2 n+\theta, n \in \mathbb{Z}, \theta \in[0,2)$. Relations (17)-(19) yield

$$
\begin{gathered}
u(x, y, 2 n+\theta)=f_{A}^{n}(w(x+\theta, y+\theta))= \\
=\left\{\begin{array}{l}
f_{A}^{n}\left(u_{0}(x+\theta, y+\theta)\right), 0 \leq y+\theta<1, \\
f_{A}^{n+1}\left(v_{0}(x-2 y-\theta+2,2-y+\theta)\right), 1 \leq y+\theta<2, \\
f_{A}^{n+1}\left(u_{0}(x+\theta-2, y+\theta-2)\right), 2 \leq y+\theta<3,
\end{array}\right.
\end{gathered}
$$

and 


$$
v(x, y, 2 n+\theta)=\left\{\begin{array}{l}
f_{A}^{n}\left(v_{0}(x-\theta, y-\theta)\right),-1 \leq \theta-y<0, \\
f_{A}^{n}\left(u_{0}(x-2 y+\theta, \theta-y)\right), 0 \leq \theta-y<1, \\
f_{A}^{n}\left(v_{0}(x-2 y+\theta-2, y-\theta+2)\right), 1 \leq \theta-y<2 .
\end{array}\right.
$$

The representation of the solution $(u, v)$ in terms of iterations of $f_{A}$ shows that the spatial-temporal evolution of the vector field $(u(x, y, t), v(x, y, t))$ depends on the dynamics of the map $f_{A}$. This allows us to analyze the qualitative behavior of the solution $(u, v)$ with time and to observe its bifurcations when the parameter $A$ changes.

The map $f_{A}: w \mapsto(1-A)^{-1}(1-w)^{2}-1$ is topologically conjugated to the map $g_{B}: w \mapsto B w(1-w)$, where $B=2(1-A)^{-1}$, the dynamics of which was studied in detail (see, e.g., $[4,6]) ; h: w \mapsto 2^{-1}(w+1)$ is a conjugating homeomorphism. This is why we will use the results concerning the properties of $f_{A}$ without special explanation. It is known [6] that there exist three types of limit behavior of orbits of the map $f_{A}$. Namely, almost all orbits are attracted either by a circle or by a cycle of intervals or by the set homeomorphic to the Cantor set. The analysis given below shows that the realization of each of these possibilities, with the exception of the case where the attractor is a cycle of period 1 or 2 , results in the spatial-temporal chaotization of the solution of problem (12)-(15): the chaotization is accompanied by forming coherent structures (vortices) of decreasing scales.

We consider the spatial-temporal behavior of the components $u(x, y, t)$ and $v(x, y$, $t)$ as the parameter $A$ takes different values. Since the initial data are periodic in $x$ with period 1, we consider the vector field $(u, v)$ only in the region $G_{0}=\{(x, y)$ : $x \in[-0,5,0,5], y \in[0,1]\}$ and the computer figures below exhibit the flow lines of $(u, v)$ in the region $\{(x, y): x \in[-0,5,0.5], y \in[0,45,1]\}$. Moreover, we confine ourselves to the moments $t=2 n, n \in \mathbb{Z}^{+}$. (when $\theta=0$ ). since in this case formulas (20) and (21) take the simplest form

$$
\begin{aligned}
& u(x, y, 2 n)=f_{A}^{n}\left(u_{0}(x, y)\right) . \\
& v(x, y, 2 n)=f_{A}^{n}\left(v_{0}(x, y)\right),
\end{aligned}
$$

and there is no principal difference between this case and the general one (when $\theta \neq 0$ ).

The initial vector field (at $t=0$ ) is regular, the flow lines (i.e., the orbits of the system $\left.\dot{x}=A(1-A)^{-1} y\left(1-A \sin ^{2} 2 \pi x\right), \dot{y}=A y \sin 2 \pi x\right)$ are given by the formula

$$
y=-\frac{1}{2 \pi} \sqrt{\frac{1-A}{A}} \operatorname{arctg}\left(\sqrt{\frac{A}{1-A}} \cos 2 \pi x\right)+\text { const }
$$

and are presented in Fig. 1.

To analyze the vector field $(u, v)$ at the moments $t=2 n, n \in \mathbb{Z}^{+}$, one must mark a "grid" in the region $G_{0}$ which is formed by curves $l_{u}^{n}$ and $l_{v}^{n}, n \in \mathbb{Z}^{+}$. such that $u(x, y, 2 n)$ and $v(x, y, 2 n)$ vanish in $l_{u}^{n}$ and $l_{v}^{n}$, respectively. There will be singular points of the field in the junctions of the "grid". A large number of singular points thus appears. which cause spatial-temporal chaotization of a vector field. By virtue of (22), the curves $l_{u}^{n}$ and $l_{v}^{n}$ are given implicitly by the following conditions

$$
\begin{array}{ll}
l_{u}^{n} & =\left\{(x, y): u_{0}(x, y)=\alpha, \alpha \in f_{A}^{-n}(0)\right\}, \quad n=0,1, \ldots . \\
l_{v}^{n}=\left\{(x, y): v_{0}(x, y)=\beta, \beta \in f_{A}^{-n}(0)\right\}, & n=0,1, \ldots .
\end{array}
$$

where $f_{A}^{-n}(0)$ is the total preimage of the origin. Thus, we have obtained two one- 
parameter families of curves ( $n$ is a parameter) and each curve consists of a finite set of continuous branches or of a countable set of branches. The limit behavior (as $n \rightarrow \infty)$ of the field $(u, v)$ can be characterized analogously with a "grid" formed by the curves

$$
\begin{aligned}
L_{u} & =\left\{(x, y): u_{0}(x, y)=\alpha, \alpha \in \partial\left\{f_{A}^{-n}(0), \quad n=0,1, \ldots\right\}\right\}, \\
L_{v} & =\left\{(x, y): v_{0}(x, y)=\beta, \beta \in \partial\left\{f_{A}^{-n}(0), \quad n=0,1, \ldots\right\}\right\},
\end{aligned}
$$

where $\partial\left\{f_{A}^{-n}(0), n=0,1, \ldots\right\}$ is the set of limit points of the set $\left\{f_{A}^{-n}(0), n=0\right.$, $1, \ldots\}$. Evidently, $L_{u}$ and $L_{v}$ are the limit curves as $n \rightarrow \infty$ (with respect to the Hausdorff metric) for the curves $l_{u}^{n}$ and $l_{v}^{n}$, respectively.

The map $f_{A}$ has two fixed points when $A \in(0,1 / 3]$ and has a fixed point and a cycle of period 2 when $A \in(1 / 3,(\sqrt{6}-1) /(\sqrt{6}+1)]$. There is no spatial-temporal chaos in these cases and so we do not consider these here. When the parameter $A$ increases, the cycles of period 4,8, etc., arise in accordance with the Sharkovsky order [6]. This causes the chaotization of the vector field $(u, v)$ both in time and in space.

1. For $A \in((\sqrt{8}-1) /(\sqrt{8}+1), 0,47941482 \ldots)$, the map $f_{A}$ has an attracting cycle of period 3, which is denoted by $\gamma=\left\{w_{1}, w_{2}, w_{3}\right\}$, and no other aturacting sets (for any $M \geq 1$, there exists at least one repelling cycle of period $M$ ). The set $D\left(f_{A}\right)=\overline{\bigcup_{n \geq 0}\left\{f_{A}^{-n}\left(\operatorname{Per} f_{A} \backslash \gamma\right)\right\}}$ (a so-called separator) is the boundary of the domain of attraction of the cycle $\gamma$. The set $D\left(f_{A}\right)$ is homeomorphic to the Cantor set and $\operatorname{mes} D\left(f_{A}\right)=0$.

For any given $n>0$, curves (23) and (24) divide the region $G_{0}$ into subregions where both component $u$ and $v$ do not change the sign (Fig. 2); there are singularities of the field $(u, v)$ at the intersection points of the curves $l_{u}^{n}$ and $l_{v}^{n}$ (at these points, $u$ and $v$ reduce to zero). To clarify the dynamics of the field with increasing $n$, note that $\left.\partial\left\{f_{A}^{-n}(0)\right\}, n=0,1, \ldots\right\}=D\left(f_{A}\right)$ in the case under consideration. Consequently, "limit" curves (25) and (26) are given by the following formulas

$$
\begin{aligned}
L_{u} & =\left\{(x, y): u_{0}(x, y)=\alpha, \alpha \in \partial\left\{f_{A}^{-n}(0), n=0,1, \ldots\right\}\right\}, \\
L_{v} & =\left\{(x, y): v_{0}(x, y)=\beta, \beta \in \partial\left\{f_{A}^{-n}(0), n=0,1, \ldots\right\}\right\},
\end{aligned}
$$

and $L_{u}$ (and $L_{v}$ ) consists of a continuum of continuous branches and, moreover, in any neighborhood of any branch of $L_{u}$ (or $L_{v}$ ), one can find another branch of $L_{u}$ (or $L_{v}$ ). Hence, a number of singular points (in the region $G_{0}$ ) grows exponentially as $n$ increases. The evolution of the vector field $(u, v)$ (near the curves $L_{u}$ and $L_{v}$ ) is accompanied by a cascade process of appearing and splitting coherent vortices up to an arbitrarily small scale: Self-similar structures, where laminar regions of the field alternate with turbulent ones, are formed by themselves in $G_{0}$; when $n$ increases, new regions of small-scale turbulence arise and, moreover, the total area of turbulent regions tends to zero (Fig. 3).

When $n \rightarrow \infty$, the behavior of the field $(u, v)$ is as follows. The curves $L_{u}$ and $L_{v}$ divide the region $G_{0}$ into open subregions $G^{*}$ (there are countably many of these subregions in $\left.G_{0}\right)$. Since the set $D\left(f_{A}\right)$ is the boundary of the domain of attraction of the cycle $\gamma$, the vector field $(u, v)$ possesses the following properties 
where

$$
\begin{aligned}
& \|u(x, y, 2 n)-\tilde{u}(x, y, 2 n)\|_{H} \underset{n \rightarrow \infty}{\longrightarrow} 0, \\
& \|v(x, y, 2 n)-\tilde{v}(x, y, 2 n)\|_{H} \underset{n \rightarrow \infty}{\longrightarrow} 0,
\end{aligned}
$$

$$
\tilde{u}(x, y, 2 n):=f_{A}^{n}\left(\omega_{f_{A}^{3}}\left(u_{0}(x, y)\right)\right), \tilde{v}(x, y, 2 n)=: f_{A}^{n}\left(\omega_{f_{A}^{3}}\left(v_{0}(x, y)\right)\right),
$$

$\omega_{g}(w)$ is an $\omega$-limit set for the orbit of the point $w$ under the map $g ;\left\|w_{1}-w_{2}\right\|_{H}:=$ $:=\Delta_{H}$ (graf $w_{1}$, graf $\left.w_{2}\right) \forall w_{1}, w_{2}: G_{0} \rightarrow[-1,1]$, and $\Delta_{H}$ is the Hausdorff distance between sets. We call the vector field $(\tilde{u}(x, y, 2 n), \tilde{v}(x, y, 2 n))$ a limit field for $(u(x$, $y, 2 n), v(x, y, 2 n))$.

The limit field $(\tilde{u}, \tilde{v})$ is periodic in time with the period 6. At any fixed moment, it takes one of three possible values $\left(w_{i}^{(3)}, w_{j}^{(3)}\right), i, j=1,2,3$, in each of the subregions $G^{*}$, because the set $D\left(f_{A}\right)$ is the boundary of the domain of attraction of the cycle $\gamma$. If we take an arbitrary subregion $G_{1}^{*}$, where $\left(\tilde{u}\left(x, y, n_{0}\right), \tilde{v}\left(x, y, n_{0}\right)\right)=\left(w_{i_{1}}\right.$, $\left.w_{j_{1}}\right)$, and an arbitrary subregion $G_{2}^{*}$, where $\left(\tilde{u}\left(x, y, n_{0}\right), \tilde{v}\left(x, y, n_{0}\right)\right)=\left(w_{i_{2}}, w_{j_{2}}\right)$ (it is possible that $\left.\left(i_{1}, j_{1}\right)=\left(i_{2}, j_{2}\right)\right)$, then there exists a subregion $G_{3}^{*}$ such that $(\tilde{u}(x, y$, $\left.\left.n_{0}\right), \tilde{v}\left(x, y, n_{0}\right)\right)=\left(w_{i_{3}}, w_{j_{3}}\right)$ and $\left(i_{3}, j_{3}\right) \neq\left(i_{1}, j_{1}\right),\left(i_{3}, j_{3}\right) \neq\left(i_{2}, j_{2}\right)$. This effect is an analog of the Serpinsky "carpet."

Note that the chaotization of the vector field $(u, v)$ follows a somewhat different course if an attracting cycle has the period $2^{k}, \forall k \geq 2$. In this case, there is no Serpinsky "carpet" effect since $D\left(f_{A}\right)$ is a countable but not continual set.

2. Let $A^{*}$ be the value of the parameter $A$ such that for any $i=0,1, \ldots$, the map $f_{A^{*}}$ has a cycle of period $2^{i}$ and has no cycles of period other than $2^{i}, i=0,1, \ldots$ $\left(A^{*}=0,43976738 \ldots\right)$. Then, as is well known, all the cycles of the map $f_{A^{*}}$ are repelling. The points of the interval $[-1,1]$, with the exception of the countable set

$$
P\left(f_{A^{*}}\right)=\bigcup_{n=0}^{\infty} f_{A^{*}}^{-n}\left(\operatorname{Per} f_{A^{*}}\right),
$$

are attracted by the set which consists of the limit points of the set $\operatorname{Per} f_{A^{*}}$ and, hence, is homeomorphic to the Cantor set. Denote this attracting set by $K$. The orbit of any point from the set $K$ is dense on $K$ and almost periodic with the almost periods 1,2 , $2^{2}, 2^{3}, \ldots$. For any point $w \in[-1,1] \backslash P\left(f_{A^{*}}\right)$, there exists the point $w^{\prime} \in K$ such that $f_{A^{*}}^{n}(w)-f_{A^{*}}^{n}\left(w^{\prime}\right) \rightarrow 0$ as $n \rightarrow \infty$. Accordingly, the region $G_{0}$ is divided by the curves

$$
\begin{aligned}
& L_{u}=\left\{(x, y): u_{0}(x, y)=\alpha, \quad \alpha \in P\left(f_{A^{*}}\right)\right\}, \\
& L_{v}=\left\{(x, y): v_{0}(x, y)=\alpha, \quad \alpha \in P\left(f_{A^{*}}\right)\right\},
\end{aligned}
$$

into open subregions (the set of these subregions is countable), in which relation (29) takes place with

$$
\begin{aligned}
& \tilde{u}(x, y, 2 n):=f_{A^{*}}^{n}\left(\bigcap_{i>0} w f_{A^{*}}^{2^{*}}\left(u_{0}(x, y)\right)\right), \\
& \tilde{v}(x, y, 2 n):=f_{A^{*}}^{n}\left(\bigcap_{i>0} w f_{A^{*}}^{2 !}\left(v_{0}(x, y)\right)\right) .
\end{aligned}
$$


Thus, the limit vector field $(\tilde{u}(x, y, 2 n), \tilde{v}(x, y, 2 n))$ is almost periodic in time (with the almost periods $\left.2,2 \cdot 2,2 \cdot 2^{2}, \ldots\right)$ and takes values from the set $\left\{\left(w^{\prime}, w^{\prime \prime}\right): w^{\prime}\right.$, $\left.w^{\prime \prime} \in K\right\}$. As in the previous case, for $t=2 n, n=0,1,2, \ldots$, there are singular points of the field $(u, v)$ at nodes of the "grid." The set $P\left(f_{A^{*}}\right)$ is an $\alpha$-limit set for any point $w \in[-1,1] \backslash P\left(f_{A^{*}}\right)$ (in particular, for $w=0$ ). Therefore, the singular points are located near by the curves $L_{u}$ and $L_{v}$ as $n$ grows and the evolution of $(u, v)$ as $n \rightarrow \infty$ is accompanied by a cascade process of emergence of structures of decreasing scales (Fig. 4).

3. When $A=1 / 2$, almost every orbit is attracted by the interval $[-1,1]$ and, consequently, it is dense on $[-1,1]$. In particular, the set $\left\{f_{A}^{-n}(0), n=0,1, \ldots\right\}$ is dense on $[-1,1]$. This is why the points $(x, y)$, at which the field $(u(x, y, 2 n), v(x, y$, $2 n)$ ) vanishes for $n=0,1, \ldots$, are everywhere dense in $G_{0}$. Hence, when $n$ increases, a cascade process of emergence of vortices of decreasing scales up to arbitrarily small ones takes place in $G_{0}$ and, in contrast to the previous cases, this process is accompanied by splitting large-scale vortices (Fig. 5). There is a complete turbulization of the field $(u, v)$ as $n \rightarrow \infty$. The limit vector field does not exist in an ordinary sense and, when time is large, we are able only to reveal the probability that $(u, v)$ takes a certain value from $[-1,1] \times[-1,1]$.

For $A=1 / 2$, the map $f_{A}$ has the invariant smooth measure with the density $\mu(d w)=d w /\left(\pi \sqrt{1-w^{2}}\right)$. This makes the statistical description of the field $(u, v)$ as $n \rightarrow \infty$ possible. The mathematical justification of the statements given below can be found in [5]. For the vector field $(u, v)$, we introduce a certain random vector field (as the limit one)

$$
\begin{gathered}
\tilde{u}(x, y):=\xi(x, y), \\
\tilde{v}(x, y):=\xi(x-2 y,-y)
\end{gathered}
$$

induced by the two-dimensional stochastic process $\xi(z, \tau)$ such that $\xi(z, \tau)$ is the random variable with the distribution function

$$
F(v)=\int_{-1}^{v} \mu(d w)=\frac{1}{\pi} \arcsin v+\frac{1}{2}
$$

for any $(z, \tau) \in \mathbb{R} \times[-1,1]$ and $\xi\left(z^{\prime}, \tau^{\prime}\right)$ and $\xi\left(z^{\prime \prime}, \tau^{\prime \prime}\right)$ are independent random variables for any $\left(z^{\prime}, \tau^{\prime}\right) \neq\left(z^{\prime \prime}, \tau^{\prime \prime}\right)$.

The evolution of the vector field $(u, v)$ as $n \rightarrow \infty$ is described by the random vector field $(\tilde{u}, \tilde{v})$ in the following sense. For any $\varepsilon>0$ and an arbitrary finite collection of points $\left(x_{i}, y_{i}\right) \in D$ and ( $\mu$-measurable) sets $A_{i}, B_{i} \in I, i=1,2, \ldots, k$, there exists an integer $N^{*}<\infty$ such that the difference between the frequency, with which the field $\left(u\left(x_{i}, y_{i}\right), v\left(x_{i}, y_{i}\right)\right)$ takes values from $A_{i} \times B_{i}, i=1,2, \ldots, k, n=0$, $1, \ldots, N$, and the probability that $\left(\tilde{u}\left(x_{i}, y_{i}\right), \tilde{v}\left(x_{i}, y_{i}\right)\right) \in A_{i} \times B_{i}, i=1,2, \ldots, k$, is at most $\varepsilon$ if $N>N^{*}$. A phenomenen of such type in deterministic systems (i.e., the behavior of a system is described by stochastic laws asymptotically accurately when time is large) is called the self-stochasticity [5].

4. Computer analysis. In this article, we pay much attention to the computer experiment, which combines modern analytical and numerical methods with a large 
ROMANENKO E. YU., VEREIKINA M. B.

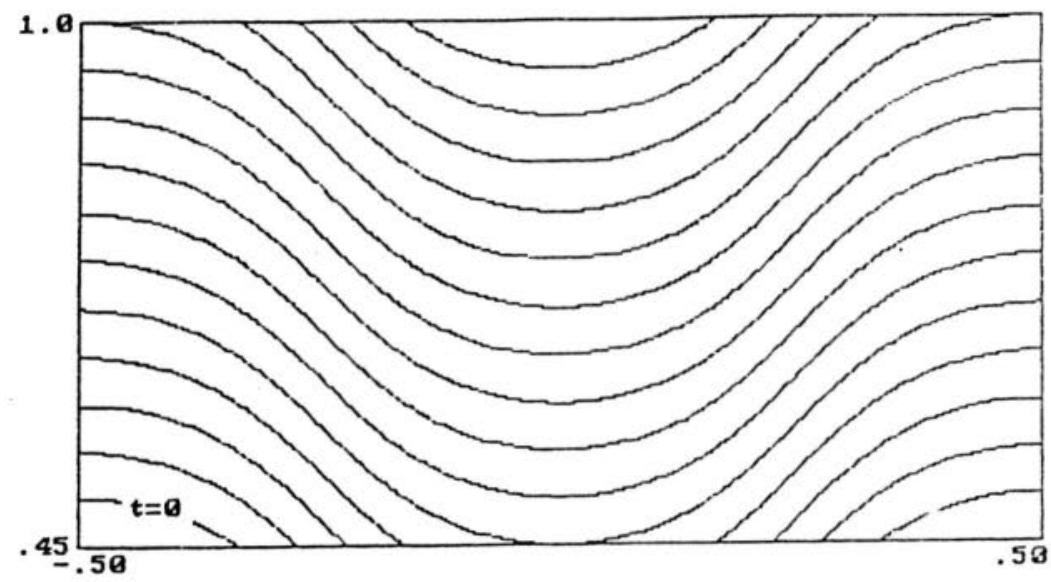

Fig. 1

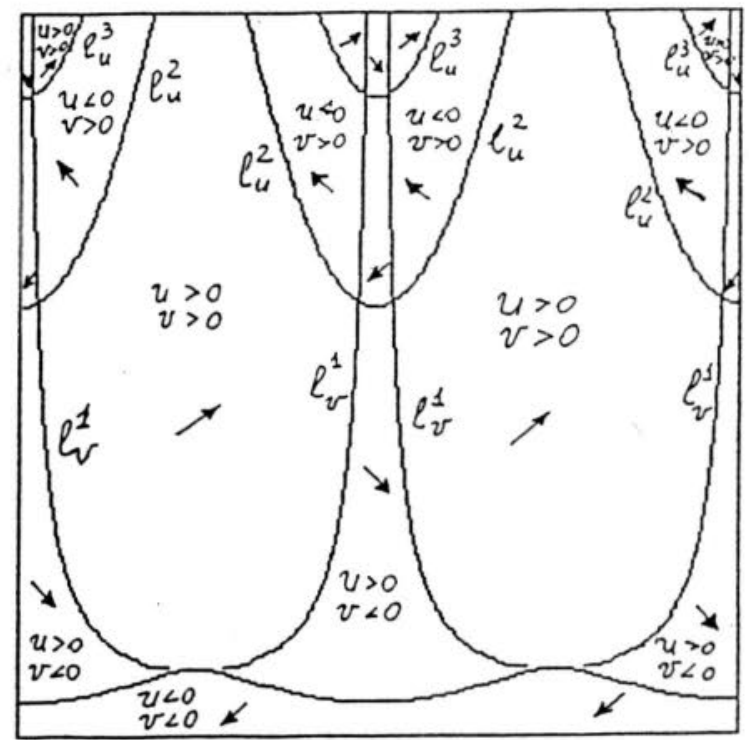

Fig. 2 
ON SIMULATION OF SPATIAL-TEMPORAL CHAOS: ...
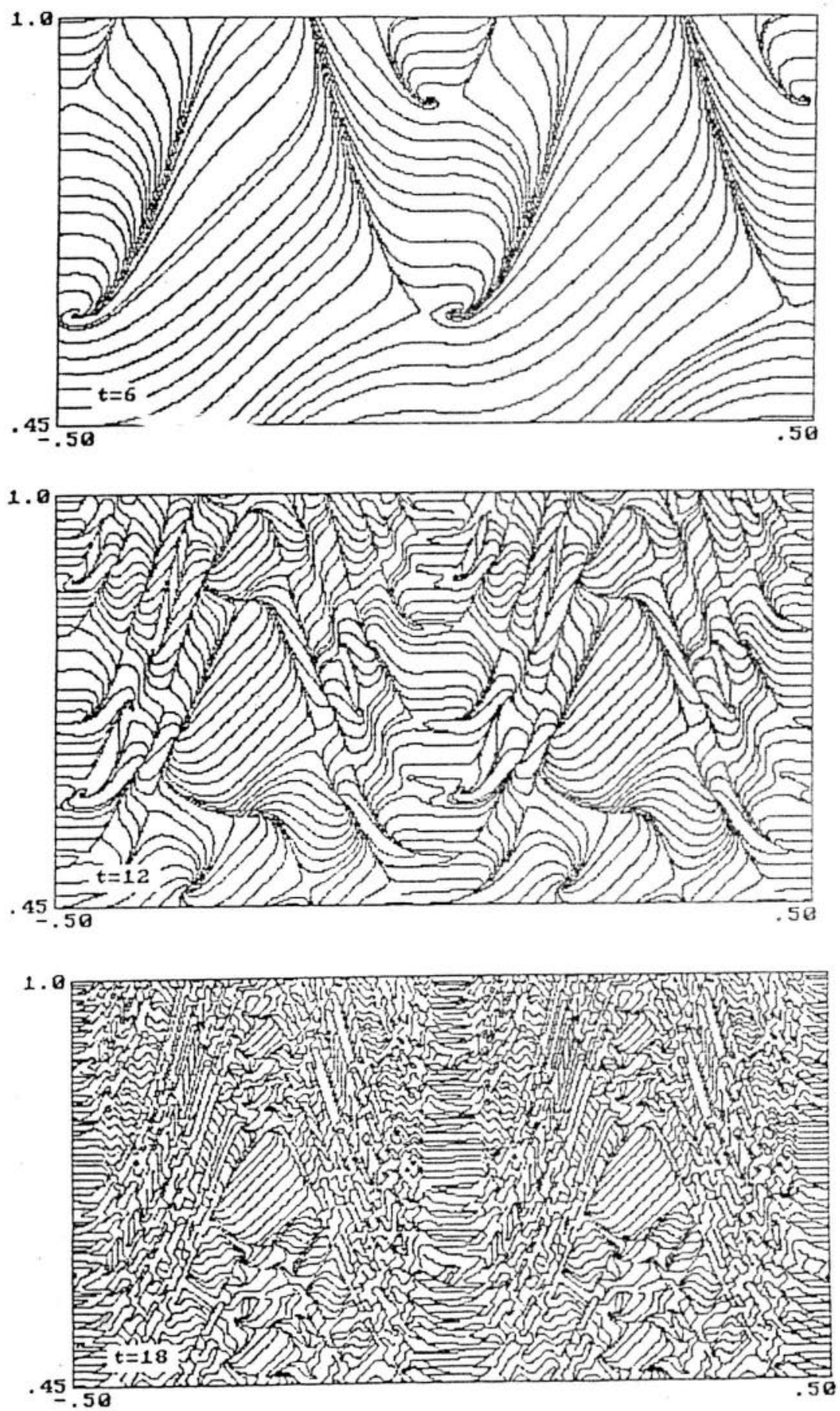

Fig. 3 

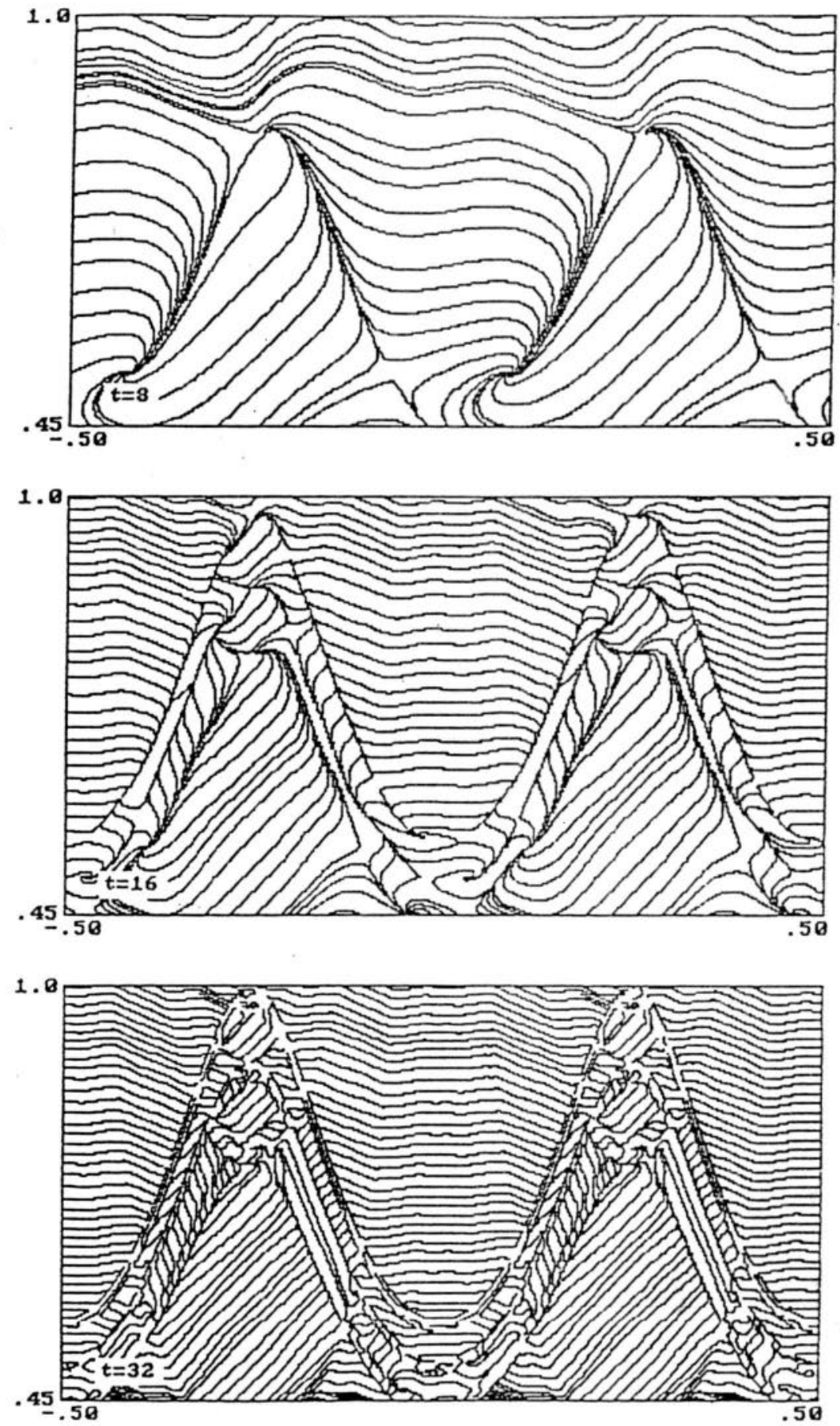

Fig. 4 

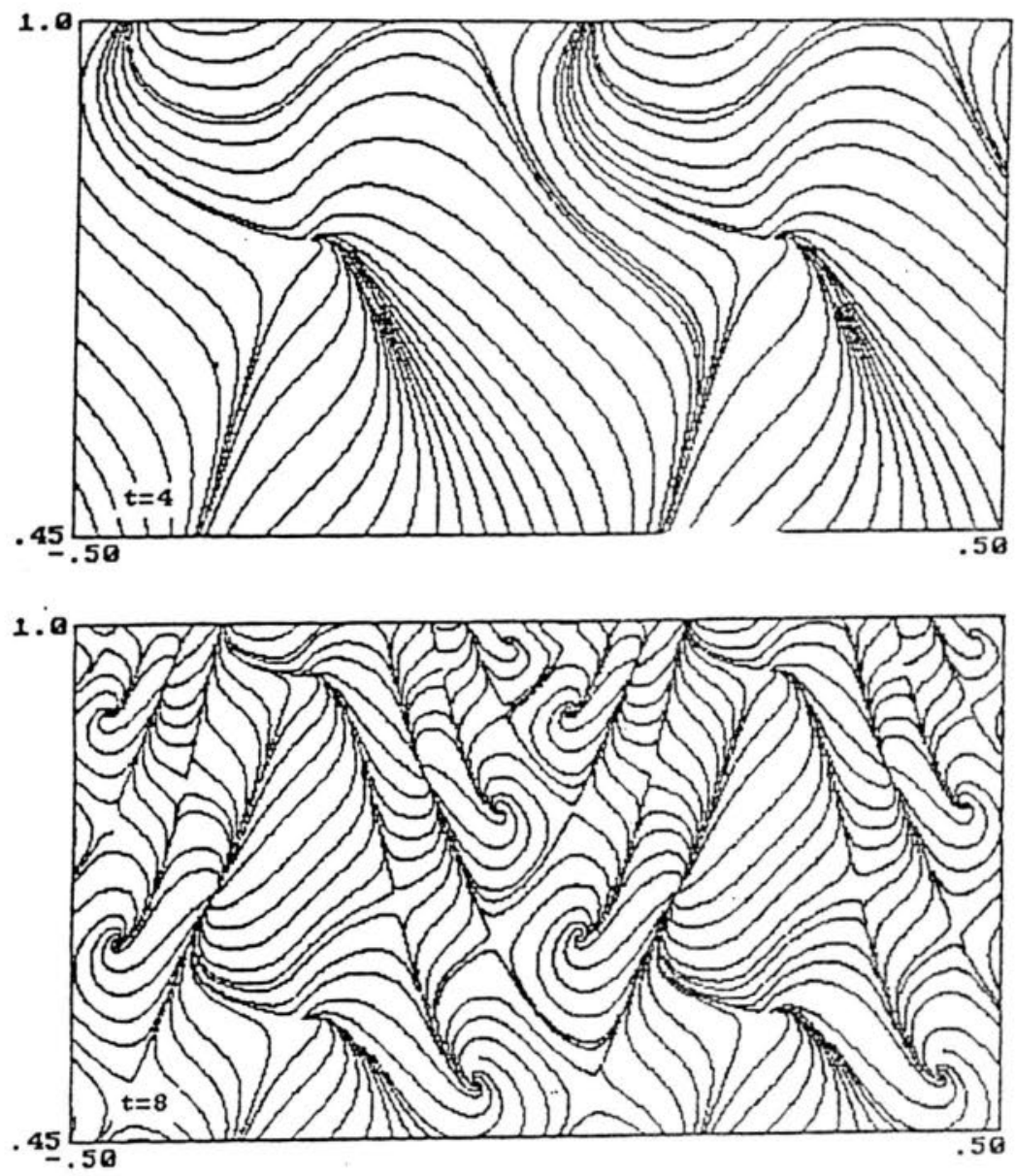

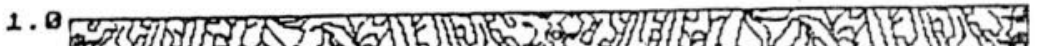
E. 910 (s)

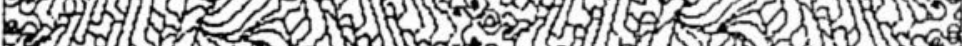
con (o)

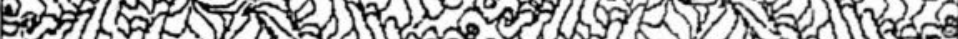

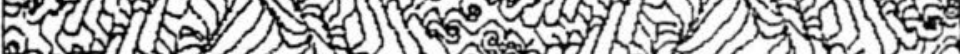
8. 4 (2)

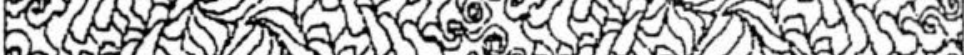

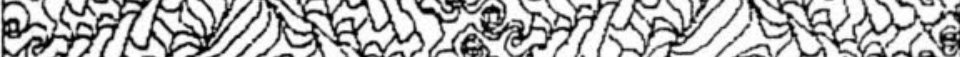
6) 30 (2)

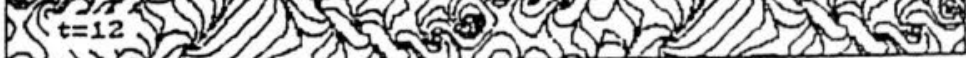
.45 ab 50 ale

Fig. 5 
amount of calculations and computer graphics. For given problem (12)-(15), the worked out software makes it possible to visualize the evolution of the vector field $(u, v)$ and the process of arising and expanding of spatial-temporal chaos.

One of the techniques for the graphic representation of a vector field consists in depicting it by using the corresponding vectors at the nodes of a spatial-temporal mesh. The vector at a node and the vector field at this node are the same in direction; the vector magnitude at a node is proportional to the vector-field magnitude at this node and the proportion factor is taken so that the magnitude of each vector is less than the half of the spatial mesh width. Such graphic representation of vector field shows the direction of motion and the relative change in velocity along the flow lines (of the vector field) at any fixed moment $t \in \mathbb{R}^{+}$but does not show the small-scale structures formed by the flow lines.

This is why we have preferred another technique of graphic representation which is based on depicting the flow lines of a vector field. In this case, if a multicolor monitor (or a multicolor printer) is available, one can visualize the velocity magnitude along a flow line. For this purpose, one should associate a certain range of velocity magnitude (as narrow as possible) with a certain color and paint a flow line according to the change of velocity. The direction of motion along a flow line may be indicated by an arrow. However, arrows obstruct the visual perception of small-scale structures.

From the viewpoint of the software realization, surely, the former of these two techniques is much_simpler. But we have preferred the latter, since it presents the graphic information in the better form.

The figures given in the article exhibit the instantaneous flow lines of the vector field $(u, v)$ at fixed moments $t^{*} \in \mathbb{R}^{+}$, i.e., the curves on the plane $(x, y)$ determined by the equations

$$
\frac{d x}{d \tau}=u\left(x, y, t^{*}\right), \frac{d y}{d \tau}=v\left(x, y, t^{*}\right) .
$$

The values of the components $u$ and $v$ at each node of the spatial-temporal mesh are found by the analytical formulas (20) and (21). Equations (33) are integrated by the simplest method (the Euler method) that gives the accuracy needed. In the developed application software, the "automated algorithm for the control in the density of flow lines". which was created while working out this software, is used for constructing flow lines.

The software has certain service resources. But we do not seek to examine them in this paper.

5. Conclusion. The examples considered show that the following three kinds of dynamic behavior are typical for boundary-value problems of form (2)-(5): asymptotically periodic, asymptotically almost periodic, and self-stochastic (i.e., asymptotically stochastic).

1. Schuster H. G. Deterministic Chaos: An Introduction. - Weinheim: Phisik-Verlag, 1984. - 240 p. (Рус. перевод: Шустер Г. Детерминированный хаос: Введение. -М.: Мир, 1988. - 240 с.)

2. Bunimovich L. A., Sinai A. G. Space-Time Chaos in Coupled Map Lattice // Nonlinearity. - 1988. $-1, N^{\circ} 4 .-$ P. $491-516$.

3. Шарковский А. Н., Майстренко Ю. Л., Романенко Е.Ю. Разностные уравнения и их приложения. - Киев: Наук. думка, 1986. - 240 с.

4. Buтm А. А. К теории скрипичной струны // Журн. техн. физики. - 1936. - 6, № 9. - С. $1459-1479$.

5. Sharkovsky A.N., Romanenko E. Yu. Ideal turbulence: attractors of deterministic systems may lie in the space of random fields // Bifurcation and Cnaos in applied sciences and engineering (World Scientific). - 1992. - 2, $\mathrm{N}^{2} 1$. - P. $31-36$.

6. Динамика одномерных отображений / А. Н. Шарковский, С. Ф. Коляда, А. Г. Сивак, В. В. Федоренко. -Киев: Наук. думка, 1989. - 216 с. 